\title{
Synthesis of oxazolones under Dakin-West conditions
}

\author{
Luciana Dalla Vechia $^{1,2}$, Raquel de Oliveira Lopes ${ }^{1,2}$, Leandro Soter de Mariz e \\ Miranda $^{1,2}$, Rodrigo Octavio Mendonça Alves de Souza ${ }^{1,2}$ \\ ${ }^{1}$ Grupo de Biocatálise e Síntese Orgânica, Instituto de Química -Universidade Federal do Rio de \\ Janeiro, CEP:22941-909 \\ ${ }^{2}$ Programa de Pós-graduação em Química, Universidade Federal do Rio de Janeiro (UFRJ) \\ *e-mail lucianadallavechia@yahoo.com.br
}

Keywords: oxazolone Dakin-West,a-amino-ketone

\section{INTRODUCTION}

The Dakin-West reaction is a known route for the synthesis of $\beta$-acetamido ketones from $\alpha$ aminoacids. The reaction takes place in the presence of carboxylic anhydrides and a base such as pyridine (Scheme 1). ${ }^{1}$

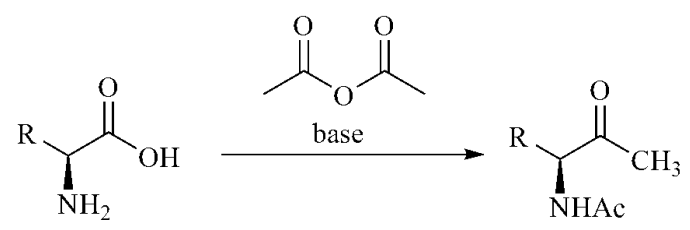

Scheme 1. Dakin-West reaction.

In the current work we report the use of chloroacetic anhydride in the Dakin-West reaction.

\section{RESULTS AND DISCUSSION}

We proceeded the reaction using phenylalanine (520 mg; $3 \mathrm{mmol})$, chloroacetic anhydride $(1,5 \mathrm{~g} ; 9$ mmol; $3 \mathrm{eq})$, methylimidazole $(0,105 \mathrm{ml}, 1,2 \mathrm{mmol}$; $0,5 \mathrm{eq}$ ) as the catalyst and dioxane as the solvent ( 5 $\mathrm{ml})^{2}{ }^{2}$ The reaction was kept under reflux during 2 hours. Afterwards, water was added to hydrolyze the remaining anhydride. The organic layer was washed with saturated solution of potassium bicarbonate and water and then dried with anhydrous $\mathrm{Na}_{2} \mathrm{SO}_{4}$. After evaporation of the solvent, the product was recrystalized in a mixture of ethyl acetate and hexane (468 $\mathrm{mg} ; 84 \%$ yield).

Surprisingly, the espectroscopical data for the isolated product indicated the absence of methylene as well as the chlorine atom. A methyl group was observed in these analysis. These data correspond with the data reported for oxazolone $\mathbf{2}$ as the sole product of the reaction.

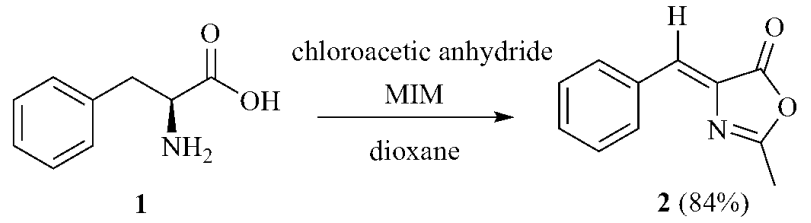

The formation of compound 2 can be explained by an overview of the reaction mechanism. The mechanism dictates that the acetyl-aminoacid 3 cyclizes to form the oxazolinone 4 through the intermediate of a mixed anhydride. In this case, further elimination of chloridric acid happens and a thermodynamic equilibrium between both tautomer takes place. Because tautomer $\mathbf{6}$ is thermodynamically more stable its formation is favoured, and this is the only product observed.

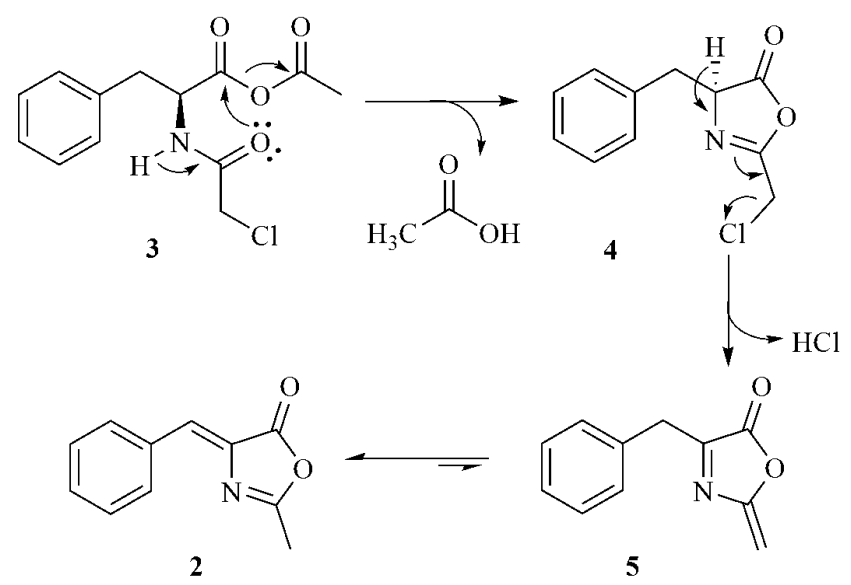

Scheme 3. Reaction mechanism overview and formation of oxazolone 2.

\section{CONCLUSION}

In summary, we synthesized oxazolone 2 in excellent yield (84\%) from phenylalanine, chloroacetic anhydride. To the best of our knowledge this is the first report of the synthesis of oxazolones under Dakin-West reaction conditions.

\section{ACKNOWLEDGEMENTS}

FINEP, CAPES, FAPERJ, CNPq and PGQUUFRJ.

\section{REFERENCES}

${ }^{1}$ Dakin, H. D.; West, R. J. Biol. Chem. 1928, 78, 91

2 Tran, K. -V.; Bickar, D. J. Org. Chem. 2006, 71, 6640.

Scheme 2. Synthesis of oxazolones under Dakin-West conditions. 